\title{
Wind Directional Effect on a Single Storey House Using Educational Wind Tunnel
}

\author{
S S Zaini ', N Rossli', T A Majid", S N C Deraman ${ }^{1}$ and N A Razak ${ }^{2}$ \\ ${ }^{1}$ Disaster Research Nexus, School of Civil Engineering, Universiti Sains Malaysia, Engineering Campus, 14300 NibongTebal, Penang \\ Malaysia \\ ${ }^{2}$ School of Aerospace Engineering, Universiti Sains Malaysia, Engineering Campus, 14300 NibongTebal, Penang, Malaysia
}

\begin{abstract}
Wind tunnel testing of single-storey isolated building with 1: 100 scale down model was carried out in an open circuit wind tunnel without roughness elements facilities. The gable roof building model with $30^{\circ}$ roof pitch was studied for wind directions of $0^{\circ}, 30^{\circ}, 45^{\circ}, 60^{\circ}$ and $90^{\circ}$.Pressure measurements were performed on all the walls and the roof (Zone 1, 2, 34 and 5) of the building model with wind speed of $12 \mathrm{~m} / \mathrm{s}$. The results showed that the high suctions were generally induced by the $90^{\circ}$ wind direction for Zone 1 and $60^{\circ}$ and $90^{\circ}$ wind directions for Zone 2 . Mostly, high suction was also observed in case of $45^{\circ}$ and $60^{\circ}$ wind direction in Zone 3 . In zone 4 and zone 5 , high suction was generally induced by the $0^{\circ}$ wind direction.
\end{abstract}

\section{Introduction}

Wind engineering covers the study on the effect of wind flow in natural environment. Moreover, this engineering discipline investigates the possible damage that may be caused by a strong wind event towards a structure, wind conform and the energy that can be extracted for mankind. High wind speed events can cause widespread destruction as reported by [1].

Most of the houses all over the world are constructed as low-rise building 1 where the mean roof height is less than the least horizontal dimension and also less than 18.3 m 2. In Malaysia, severe damage on low-rise building always occurred in the rural area especially in the northern region of Peninsular Malaysia due to windstorm events. Most of the damages occurred on the roofing system, caused by high local suctions and large pressure fluctuations around the roof structure 3 .

Wind pressure may not necessarily exert to the surface of the building wall at $0^{\circ}$ as it can vary at different angles and the pressures are dependent on the wind direction 4. When the approaching wind is normal to the ridge, the windward wall and the leading edge of the sidewalls experience high pressure and high suctions, respectively. Holmes 5 studied the wind flow on a single-storey house with $10^{\circ}$ pitch roofs using wind tunnel facilities. The wind directions were set at $0^{\circ}, 60^{\circ}$ and $90^{\circ}$. The results showed that the $60^{\circ}$ wind direction produced the critical mean suction on the gable roof due to conical vortex along the edges and corner of the roof.

Jing and $\mathrm{Li} 6$ investigated the wind pressure distribution on a 1:40 scale low-rise house model using wind tunnel facilities. They reported that for $0^{\circ}$ wind direction, the largest absolute values of peak negative wind pressure occurred on the edges of windward wall, the leading edges of windward roof and the leeward roof ridge. In the case of $45^{\circ}$ wind direction, the largest absolute values of peak negative wind pressure occur on the corner and edge of the windward wall, the windward roof corner, and the leeward roof ridge due to the formation of conical vortex and separation of airflow.

The number of houses being damaged during windstorm events increased annually in Peninsular Malaysia This phenomenon becomes worse where the surrounding economic activities are mainly agriculture. Such locations are large area of flat land for paddy plantation. This type of open terrain provides no obstacle to shield the wind flow.

The low-rise buildings in rural area are more susceptible during monsoon seasons which compared to that in the urban area where wind flow is commonly obstructed or shielded by other building structures. In an open area, wind can impinged on a building from any direction and as such, there is a need to study the effect of wind flow from several angles.

This study aims to investigate the distribution of the pressure coefficients along the profile of a down scale isolated low rise building model using wind tunnel test facilities. All tests pertaining to the effect of wind direction conducted by previous researchers used boundary layer wind tunnel test facilities. Where the model is a scaled single storey house model, there is a possibility to use educational wind tunnel test without using roughness element. This approach reduces the experimental time and cost. 


\section{Experimental setup and procedure}

The tests were conducted using an open circuit wind tunnel or Eiffel wind tunnel. The wind tunnel has a working section of $300 \mathrm{~mm}$ wide, $350 \mathrm{~mm}$ high and 550 $\mathrm{mm}$ long as shown in figure 1 . This type of tunnel has a very low turbulence level, approximately $0.15 \%$. All tests were performed at wind speed of $12 \mathrm{~m} / \mathrm{s}$.

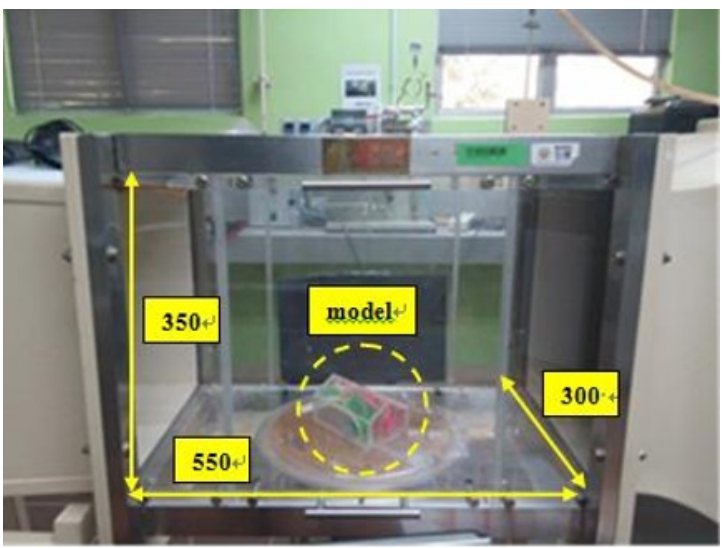

Figure 1.Test section (all dimensions in $\mathrm{mm}$ ).

The building model was fabricated using $6 \mathrm{~mm}$ thick Perspex. The dimension for model is $80 \mathrm{~mm}$ width, 120 $\mathrm{mm}$ length and $40 \mathrm{~mm}$ height following 7 . The locations of the pressure taps placed on the walls and roofs of the models are shown in figure 2. The blockage ratio for the models in the wind tunnel ranged from 2.62 to $7.21 \%$. $100 \mathrm{~cm}$ long PVC tube with $0.1 \mathrm{~cm}$ internal diameter and $0.2 \mathrm{~cm}$ external diameter were inserted into the holes drilled into the Perspex. The model was placed on top of the turntable located at the middle of the test section. Each pressure tap on the building model was connected to differential pressure sensor. The channel box allowed simultaneous monitoring of 8 pressure tap readings. The measured values of static pressure were converted to pressure coefficient, $\mathrm{Cp}$, which is defined as

$$
C_{P}=\frac{P-P_{\infty}}{0.5 \rho U_{\infty}^{2}}
$$

$\mathrm{P}$ is the pressure at a given location of model, $P_{\infty}$ is the free stream static pressure, $U_{\infty}$ is the free stream velocity and $\rho$ is the air density. Since the wind tunnel is able to generate wind in $0^{\circ}$ only, the assignment of $30^{\circ}$, $45^{\circ}, 60^{\circ}$ and $90^{\circ}$ directions were done by turning the model to the desired angle.

\section{Results and discussion}

Figure 3 compares the results between Ozmen et al. 7 and present study. It can be seen that the use of educational wind tunnel facilities is able to produce similar trend along the profile of roofs and walls. The differences in the results occurred especially at the end of wall $\mathrm{A}$ and leading edge of roof B. However, the disparity is due to the location and quantity of the pressure taps. The present study needs to maintain several pressure tap location as required by the following test. The ability of the educational wind tunnel facilities to produce reliable results was extended to the investigation on the effect of wind direction.

Figure 4 shows the pressure coefficient along the walls and roofs for wind directions $0^{\circ}, 30^{\circ}, 45^{\circ}, 60^{\circ}$ and $90^{\circ}$ in Zone 1 . In the case of $0^{\circ}$ wind direction, high pressure was developed on the windward wall (region A) where the maximum positive pressure coefficient was recorded to be 0.96 . However, the pressure coefficient reduced as it approaches the leading edge of the wall and converted to suction in region $\mathrm{B}$ with maximum suction of -0.89 . The change from pressure in region $\mathrm{A}$ to suction in region B is because the air flow has already separated at the leading edge of the windward roof forming a separation zone 6 . In region $\mathrm{C}$ and $\mathrm{D}$, the suction remains almost constant with an average reading of -1.44 .

Figure 4 shows the pressure coefficient along the walls and roofs for wind directions $0^{\circ}, 30^{\circ}, 45^{\circ}, 60^{\circ}$ and $90^{\circ}$ in Zone 1 . In the case of $0^{\circ}$ wind direction, high pressure was developed on the windward wall (region A) where the maximum positive pressure coefficient was recorded to be 0.96 . However, the pressure coefficient reduced as it approaches the leading edge of the wall and converted to suction in region $B$ with maximum suction of -0.89 . The change from pressure in region $\mathrm{A}$ to suction in region B is because the air flow has already separated at the leading edge of the windward roof forming a separation zone 6 . In region $\mathrm{C}$ and $\mathrm{D}$, the suction remains almost constant with an average reading of -1.44 .

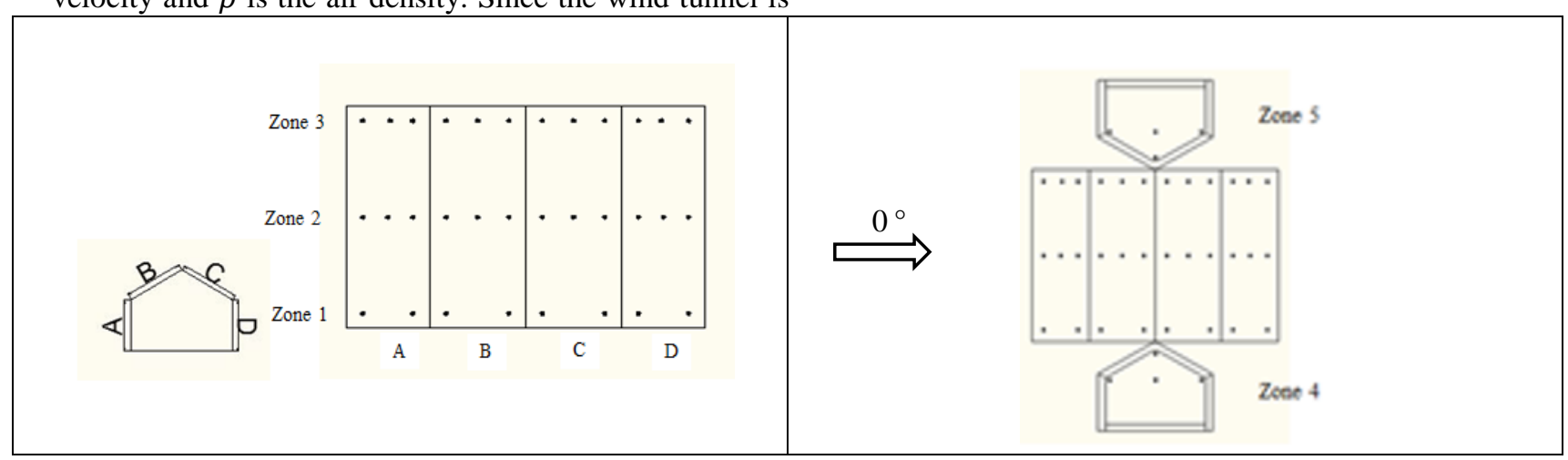

Figure 2. The building model showing the location of pressure taps for Zone 1 to Zone 5 


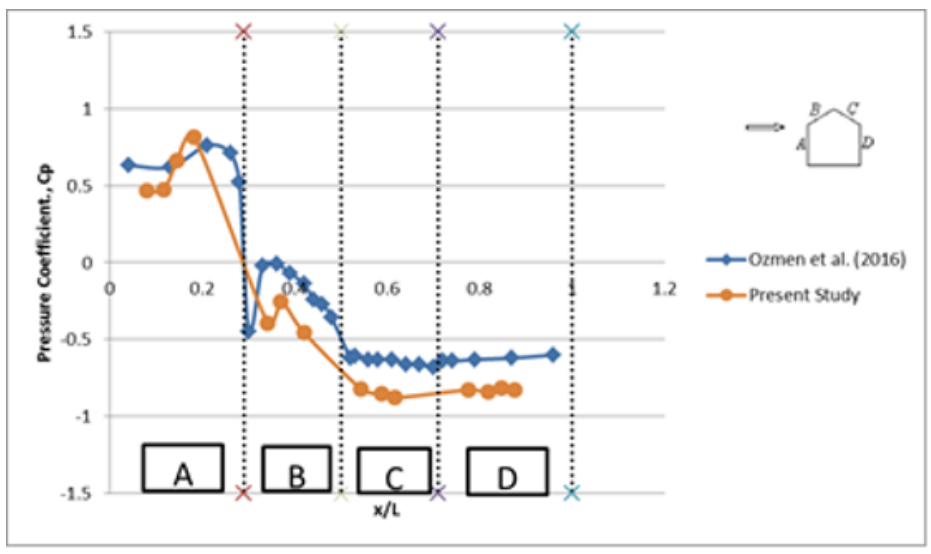

Figure 3.Comparison between Ozmen et al. [8] with present study.

The same trend was also observed for $30^{\circ}$ and $45^{\circ}$ wind directions. However, the positive pressure coefficient at the lower end of the wall in region $\mathrm{A}$ was higher (approximately 0.91) compared to the $0^{\circ}$ wind direction (0.30). On the contrary, the pattern of the pressure coefficient in region $\mathrm{A}$ for $60^{\circ}$ wind direction immediately reduced as it approaches the leading edge of the roof and converted to suction with a maximum value of -2.18 in region $\mathrm{B}$. The suction decreased in region $\mathrm{C}$ and maintained almost the same magnitude in region $\mathrm{D}$. In the case of $45^{\circ}$ wind direction, the wind impinged the model at a skewed angle. As a result, conical vortex was formed at the leading edge and created suction in region $\mathrm{B}, \mathrm{C}$ and $\mathrm{D}$. As for the $30^{\circ}$ and $60^{\circ}$ wind direction, the conical vortex was developed at the roof corner.

Finally, in the case of $90^{\circ}$ wind direction, the distribution of the pressure coefficient changed drastically where all the surfaces only exhibited negative pressure coefficients 6 . The highest suction showed by the $90^{\circ}$ wind direction at the leading edge of roof in region $\mathrm{B}$ can be associated to the formation of separation point at that particular area leading to the development of separation zone 8. Similarly, the reduction in the negative pressure coefficient observed in region $\mathrm{C}$ and $\mathrm{D}(-2.07)$ can be related to the reattachment process of the separation zone occurred as the wind approached the leeward surfaces as reported by 9 .

Figure 5 shows the pressure coefficient for wind speed at $12 \mathrm{~m} / \mathrm{s}$ with the variation of wind directions $0^{\circ}, 30^{\circ}$, $45^{\circ}, 60^{\circ}$ and $90^{\circ}$ in Zone 2 . In the case of $0^{\circ}$ wind direction high pressure was occurred on the windward wall (region A) where the maximum positive pressure coefficient was recorded to be 0.76 . However, the pressure coefficient decreased as it approaches the leading edge of the wall and converted to suction in region $B$. It can be seen that the magnitude of the suction slightly fluctuated. The maximum suction in region $\mathrm{B}$ showed to be approximately $(-0.84)$. In region $\mathrm{C}$ the suction remains almost constant with an average reading of (-1.43). This occurrence continues in region D. In general, the same trend was observed as in the case of $0^{\circ}$ wind direction in Zone 1.

The same trend was also observed for $30^{\circ}$ and $45^{\circ}$ wind directions. However, the positive pressure coefficient at the lower end of the wall in region A was higher (approximately 0.53 ) compared to the $0^{\circ}$ wind direction (0.44). The suction increased slightly in region $\mathrm{C}$ and remained almost the same magnitude in region $\mathrm{D}$. The higher suction showed in region $\mathrm{C}$ for $60^{\circ}$ wind direction can be related to the formation of larger separation zone closer to region $\mathrm{C}$.

Finally, in the case of $90^{\circ}$ wind direction, the distribution of the pressure coefficient changed drastically where all the surfaces only exhibited negative pressure coefficients (suction). The highest suction showed by the $90^{\circ}$ wind direction is located at the ridge of roof in region C (-1.80).

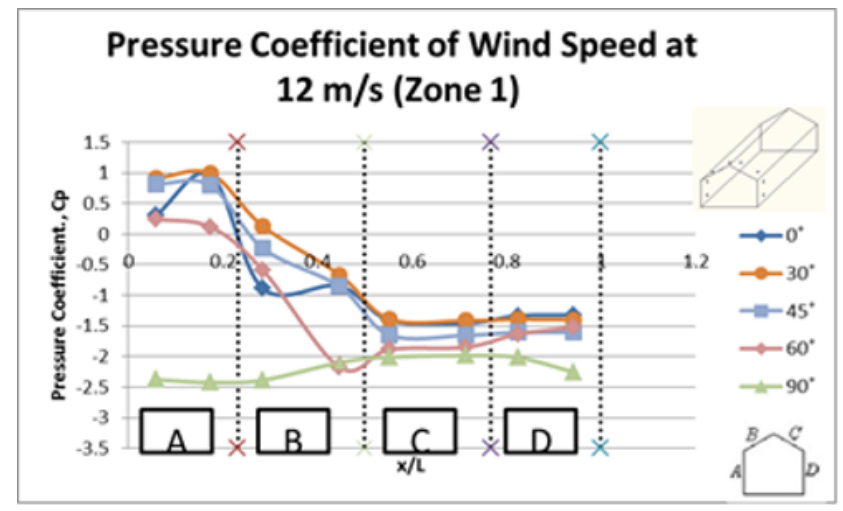

Figure 4.Pressure coefficient of wind speed at $12 \mathrm{~m} / \mathrm{s}$ (Zone 1).

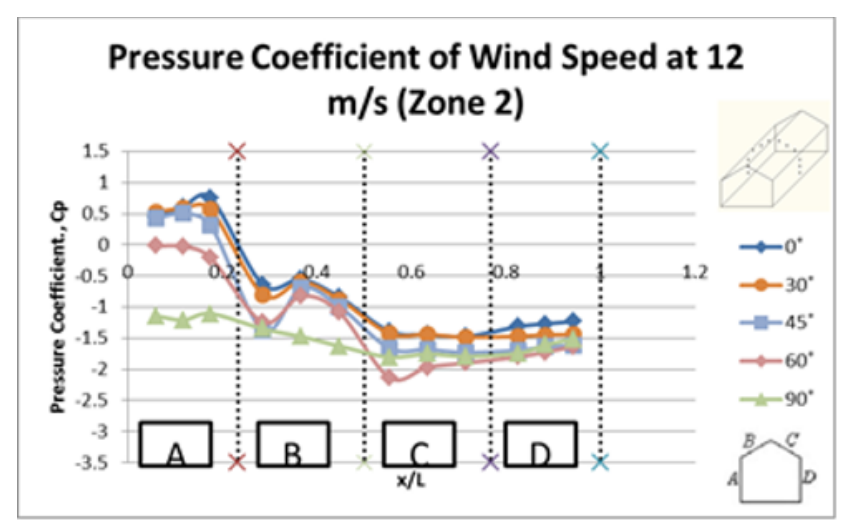

Figure 5.Pressure coefficient of wind speed at $12 \mathrm{~m} / \mathrm{s}$ (Zone 2). 
Figure 6 shows the pressure coefficient for wind speed at $12 \mathrm{~m} / \mathrm{s}$ with the variation of wind directions $0^{\circ}, 30^{\circ}$, $45^{\circ}, 60^{\circ}$ and $90^{\circ}$ in Zone 3 . In the case of $0^{\circ}$ wind direction, high pressure was developed on the windward wall (region A) where the maximum positive pressure coefficient was recorded to be 1.01. However, the pressure coefficient reduced as it approaches the leading edge of the wall and converted to suction in region $\mathrm{B}$ (maximum suction -1.02). In region $\mathrm{C}$ and $\mathrm{D}$, the suction remained almost constant with an average reading of 1.45. The same trend was also observed for $30^{\circ}$ and $45^{\circ}$ wind directions. However, the positive pressure coefficient in region A was lower (approximately 0.50) compared to the $0^{\circ}$ wind direction (0.99). The $90^{\circ}$ wind direction showed similar trend with Zone 1 and Zone 2. The high suction was located at the middle of roof in region $\mathrm{C}$ and remained constant with an average reading of -0.81 in region $\mathrm{D}$.

Figure 7 shows the pressure coefficient for wind speed at $12 \mathrm{~m} / \mathrm{s}$ with the variation of wind directions $0^{\circ}, 30^{\circ}$, $45^{\circ}, 60^{\circ}$ and $90^{\circ}$ for Zone 4 and Zone 5. In Zone 4, the positive pressure coefficients occurred in $45^{\circ}, 60^{\circ}$ and $90^{\circ}$ wind directions. In the case of $60^{\circ}$, high pressure was developed on the leading edge of wall (tap 2) as reported where the maximum pressure coefficient was recorded to be 0.69 . Similar trend was observed as in the case of $45^{\circ}$ wind direction. In the case of $90^{\circ}$ wind direction, the high pressure occurred on the middle of wall with maximum pressure coefficient was recorded to be 0.77 .

Hence, in the case of $0^{\circ}$ and $30^{\circ}$ wind directions, the distribution of the pressure coefficient changed drastically where all the surfaces only exhibited suction. The highest suction showed by $0^{\circ}$ wind direction at the leading edge of wall (tap 2) with a maximum value of -1.13 . This can be associated to the fact that airflow already separated at the leading edge of the windward wall forming a separation zone. In the case of $30^{\circ}$ wind direction, the high suction occurred at the middle of wall (tap 3$)$.

Finally, in Zone 5, suction occurred in the case of $0^{\circ}$, $30^{\circ}, 45^{\circ}, 60^{\circ}$ and $90^{\circ}$ wind directions. The highest suction showed by the $0^{\circ}$ wind direction at the leading edge of windward wall (-1.78). Similar trend was also observed as in Zone 4. In general, high suction was developed at the edge of wall. This is particularly true in all cases except for $0^{\circ}$ and $90^{\circ}$ wind directions.

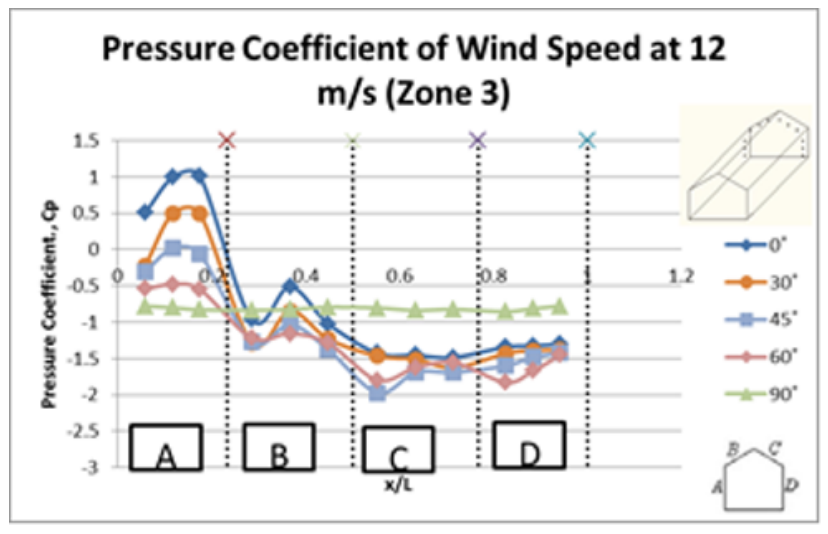

Figure 6.Pressure coefficient of wind speed at $12 \mathrm{~m} / \mathrm{s}$ (Zone 3).

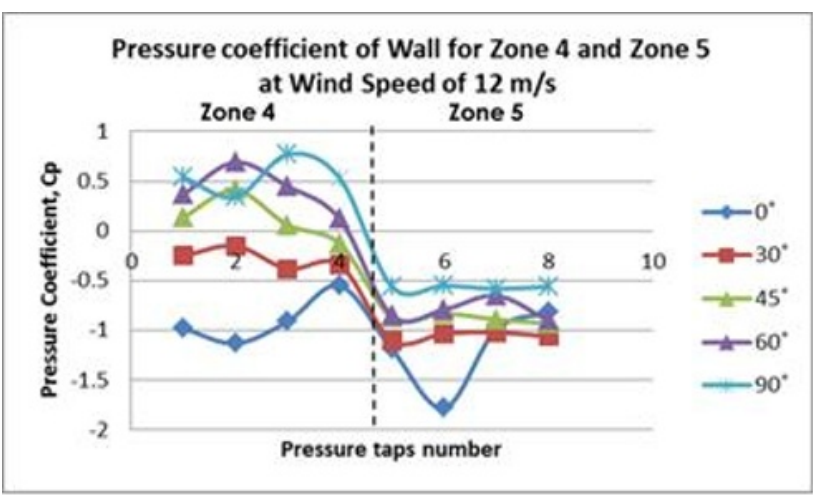

Figure 7.Pressure coefficient of wind speed at $12 \mathrm{~m} / \mathrm{s}$ (Zone 4 and Zone 5).

\section{Conclusion}

The positive pressure coefficients were recorded in region A for all wind directions except for $90^{\circ}$ wind direction in Zone 1 . Suction in region B, C and D was developed for all wind directions. The suction decreased in the case of $30^{\circ}$ wind direction and increased in the case of $45^{\circ}, 60^{\circ}$ and $90^{\circ}$ wind direction for all regions. In Zone 2, most of the suctions were lower compared to Zone 1 in region $\mathrm{B}$, $\mathrm{C}$ and $\mathrm{D}$ for $0^{\circ}, 45^{\circ}$ and $90^{\circ}$ wind direction. However, the overall distribution of the pressure coefficient was found to be relatively similar to Zone 1 . On the contrary, for Zone 3, the positive pressure coefficients were recorded in region $\mathrm{A}$ for $0^{\circ}$ and $30^{\circ}$ wind directions only. The suction increased in the case of $30^{\circ}$ wind direction and increased in the case of $45^{\circ}$ and then decreased again, in the case of $60^{\circ}$ and $90^{\circ}$ wind directions. In Zone 4 , the positive pressure coefficients were recorded for $45^{\circ}, 60^{\circ}$ and $90^{\circ}$ wind directions. Suction was developed for $0^{\circ}$ and $30^{\circ}$ wind directions. The high suction occurred at one of the edge of wall for $0^{\circ}$ wind direction and at the middle of wall for $30^{\circ}$ wind direction. High suction occurred at the edge of wall for Zone 5 in the all cases except for $30^{\circ}$ and $90^{\circ}$ wind directions.

\section{Acknowledgments}

The authors express their gratitude for the financial support of the Fundamental Research Grant Scheme (FRGS) from the Ministry of Higher Education Grant (203.PAWAM.6071317), Universiti Sains Malaysia and MyBrain15 Scholarship from the Ministry of Education Malaysia and School of Aerospace Engineering, USM for the wind tunnel laboratory.

\section{References}

1. Prasad D, Uliate T and Ahmed M R 2009 Wind loads on low-rise building models with different roof configurations Int. J. Fluid Mech. Res 36pp 231-243

2. American Society of Civil Engineers (ASCE) Standard 2002Minimum design loads for buildings and other structuresNew York, USA 
3. Ramli N I, Majid T A, Ali M I, Saad M S H, Hashim $\mathrm{M}$ and Zakaria I 2010Wind related disaster risk reduction activities in Malaysia

4. Meecham D, Surry D and Davenport A G 1991The magnitude and distribution of wind-induced pressures on hip and gable roofsJ. Wind Eng. Ind. Aerodyn.38pp 257-272

5. Holmes J D 1994 Wind pressures on tropical housing J. Wind Eng. Ind. Aerodyn.53pp 105-123

6. Jing $\mathrm{X}$ and $\mathrm{Li} \mathrm{Y}$ 2013Wind tunnel tests for wind pressure distribution on gable roof buildings Sci. World J. 2013

7. Ozmen Y, Baydar E and van Beeck J P A J 2016 Wind flow over the low-rise building models with gabled roofs having different pitch anglesBuild. Environ95pp 63-74

8. Liu H 1991Wind Engineering A Handbook for Structural Engineers New Jersey: Prentice-Hall, Inc,

9. Holmes J D 2001 Wind Loading of Structures, Second Edi London and New York: Taylor \& Francis 